\title{
Human talent forecasting
}

\author{
Bogdan NEDELCU \\ University Politehnica of Bucharest, Bucharest, Romania \\ bogdannedelcu@hotmail.com
}

\begin{abstract}
The demand for talent has increased while the offer has declined and these worrying trends don't seem to show any sign of change in the near future. According to Bloomberg Businessweek, USA, Canada, UK, and Japan (among many others) will face varying degrees of talent shortages in almost every industry in the coming years. The performed study focuses on identifying patterns which relates to human skills. Recently, with the new demand and increasing visibility, human resources are seeking a more strategic role by harnessing data mining methods. This can be achieved by discovering generated patterns from existing useful data in HR databases. The main objective of the paper is to determine which data mining algorithm suits best for extracting knowledge from human resource data, when in it comes to determining how suited is a candidate for a specific job. First of all, it must be determined a way to evaluate a candidate as objective as possible and rate the candidate with a mark from 0 to 10. To do so, some data sets had to be generated with different numbers of values or different values and wore processed using Weka. The results had been plotted so that it would be easier to interpret. Also, the study shows the importance of using large volumes of data in order to take informed decisions has recently become extremely discussed in most organizations. While finances, marketing and other departments within a company receive data systems and customized analysis, human resources are still not supported by expert systems to process large data volumes. The software prototype designed for the experiment rates individuals (working for the company, or in trials) on a scale from 0 to 10, offering the decision makers an objective analysis. This way, a company looking for talent will know whether the person applying for the job is suited or not, and how much the hiring will influence the overall rating of the department.
\end{abstract}

Keywords: human resources, talent management, data mining, big data.

\section{Introduction}

With big data's emergence, human resources (HR) is facing an unprecedented opportunity to become more focused on data, analytical and strategic in the way that talent is acquired. Indeed, HR spent much of the past decade trying to find ways of becoming better by applying metrics and analysis of human capital decisions.

Now, new and powerful technologies make it possible for HR to mix its internal data with unprecedented amounts of data from external sources to make talent management decisions based on evidence and raise the profile of the department as a strategic partner for superior leadership. This is especially true when it comes to job portals, which continues to be a source of top for employers in an attempt to find candidates for the jobs offered.

In human resources, data knowledge extraction techniques used are focused on personnel selection, especially for choosing candidates for a job. Classification and data mining prediction for human resource issues are rare and there are several examples, such as to predict the duration of a service, the first sales, persistence indices of insurance agents and operators working behavior analysis.

Since launching [MCKa], his predictions started coming true. The demand for talent has increased while the demand has declined and these worrying trends doesn't seem to 
show any sign of change in the near future. According to Bloomberg Businessweek, USA, Canada, UK, and Japan (among many others) will face varying degrees of talent shortages in almost every industry in the years 2020-2030 [BLM].

Predictions made by HR directors shows a certain pressure on the labor market in many professions, generated by the lack of staff for the 2020-2030 period.

Although the global recession has forced many organizations to resort to layoffs and focus less on talent shortages in the short term, uncertainties on the world market gave directors more reason to worry about the talent they have and the talent they need. This materialized in an intensification of interest in the human capital, which gave rise to the term of integrated management of human resources (Integrated Talent Management). Thus, HR should be integrated into a continuous cycle of actions (like forecasting, recruitment, implementing, developing, retaining human talent) that feed into each other and form an overview of the human capital of an organization, which then form business strategies and human capital.

Though the term of integrated management of human resources began to take shape, it appears that current practice has not made this step. Most companies still have a fairly isolated HR function with software utilities that desperately attempt to support, but unfortunately there are only making data analysis difficult, if not impossible. The prototype presented in the paper can be used by future HR systems developers, making it easier for managers to rake their employees and to see the impact of a candidate on the overall performance of the company.

A recent study [ADP15], highlighted in Figure 1, conducted on a sample of 725 multinational companies with over 5,000 employees worldwide, showed that on average, companies are trying to use 33 payroll systems and 31 systems for human resources management. Moreover, these numbers have increased by $40 \%$ in the last year. Thus, we can say that it is necessary to standardize company-wide management systems, by implementing an intelligent system that will help achieve managerial functions within the organization. A standard system would ease implementation of an internal mobility of employees, further exploiting the qualities of an employee by relocating to another station to provide better yield. It also would allow employees to consider a new career in another department of the company, before opting for an offer of employment from the outside. This helps to keep employees in the company, leading to a decrease in investments in new employee specialization. The prototype can be used to see if a candidate or an employee can perform better on a different role in the company.

The paper evaluates the accuracy of the following classification algorithms: random forest, $\mathrm{K}^{*}$ Star, multi-layer perception, radial basis function network or C4.5. After determining the algorithm that suits best the data set used in the case study, a prototype based on the algorithm is proposed. 


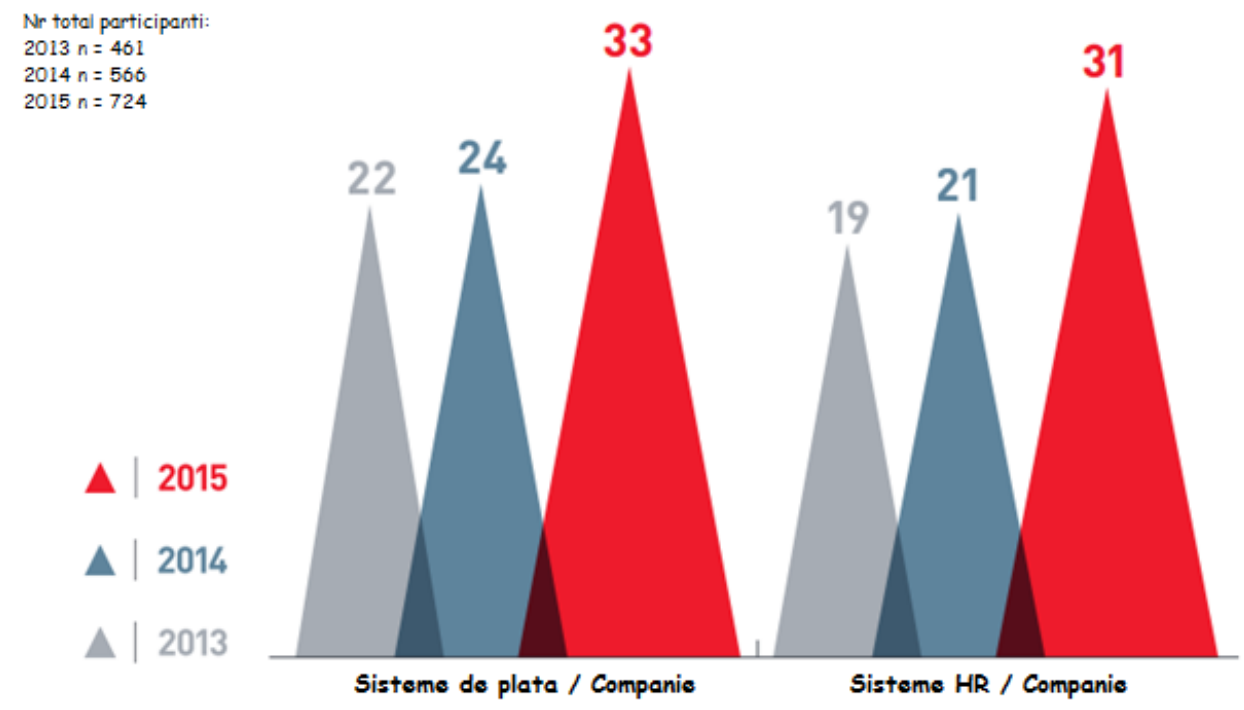

PICBE | 439

Figure 1. Data mining tasks for Talent Management

Source: ADP Research Institute: Harnessing Big Data: The Human Capital Management Journey to Achieving Business Growth - ADP Global Human Capital Management Decision Makers Survey, 2015.

The prototype can be used in HR system to rate every employee or potential candidates and rake them, offering a scoreboard to the managers. Such a classification can be used by managers to evaluate whether a candidate can improve the overall performance of the department (and with how much, precisely), or if an employee can perform better on a different job role.

\section{Data classification techniques}

Classification and prediction in data mining are two forms of data analysis that can be used to extract models for describing important data classes or to predict future data trends [HJKM]. The classification has two phases: the first phase is a learning process in which the training data is analyzed by a classification algorithm. Thus, the learned or classified model is represented as classification rules. Next, the second phase is the process of classification, where test data is used to estimate the accuracy of the classification model or classifier.

Moreover, the techniques that are used for data classification are decision trees, Bayesian methods, Bayesian network algorithms based on rules, neural networks, support machine vectors, rules of mining association, nearest neighbor $\mathrm{k}-\mathrm{NN}$, reasoning based on case, genetic algorithms, rough sets and fuzzy logic.

Further in the paper, I have used three main classification techniques, namely decision trees, neural networks and nearest neighbor k-NN. I chose these algorithms because the decision tree and neural networks are found useful in the development of predictive models in many areas. This is further supported by similar studies [TSO].

The advantages of the decision tree are that it does not require any knowledge of art or setting parameters, and is suitable for knowledge discovery exploration.

The second method is the neural networks that have high tolerance for noise data and the ability to classify a pattern that they have not been trained. This technique can be used when we have little knowledge about the relationship between attributes and classes. 
Further, the nearest neighbor k-NN technique is an example based learning, which uses the distance metric to measure the similarity courts.

There are some studies that show a great interest in solving the HR problems by extracting knowledge from data using [RANJ]. Table 1 shows some of the tasks that HR uses data mining for. In addition, until this now there is a very limited discussion on talent management, such as talent forecasting, career planning and talent recruitment through an approach that uses knowledge extracted from data.

In human resources, knowledge extraction techniques used in data focuses on personnel selection, especially for choosing candidates for a job. Classification and data mining prediction for human resource issues are rare and there are several examples, such as to predict the duration of a service, the first sales, persistence indices of insurance agents and operators working behavior analysis [CHN]. For these reasons, this paper attempts to use classification techniques for extracting knowledge from data to achieve a forecast of potential employees, showing how suitable they are for workloads that awaits them, using past experience

Table 1. - Data mining techniques

\begin{tabular}{|l|l|}
\hline & Extracting knowledge from data techniques \\
\hline Staff selection & $\begin{array}{l}\text { Decision Trees [CHN] } \\
\text { Fuzzy logic and extracting knowledge from data [TAHS] } \\
\text { Rough set theory [CCWL] }\end{array}$ \\
\hline Employee training & Association rule mining [CCWL] \\
\hline Employee development & $\begin{array}{l}\text { Fuzzy logic and artificial neural networks [HTL] } \\
\text { Decision Trees [THCS] }\end{array}$ \\
\hline Performance evaluation & Decision Trees [ZHAO] \\
\hline
\end{tabular}

Source: Author's own research.

\section{Case study}

In this paper, I will focus on one of talent management's challenges, specifically that of identifying the existing talent in terms of key talent in an organization by anticipating performance using previous records of employee performance from databases. In this case, I will use past employee in terms of their talent by using the classification techniques for mining knowledge from data.

The models can be generated using some of the most important data mining techniques, such as grouping the list of employees with similar characteristics to Group performance and other. Technical Association of models that are discovered can be used to assign the most appropriate employee profile program / job role, associated with employee attitude towards performance and other. Stage prediction and classification model discovered can be used to predict the percentage accuracy of performance, behavior and attitudes of employees, to predict the evolution of performance throughout the period of performance and also identify the best profile for different employees (Fig. 2). 


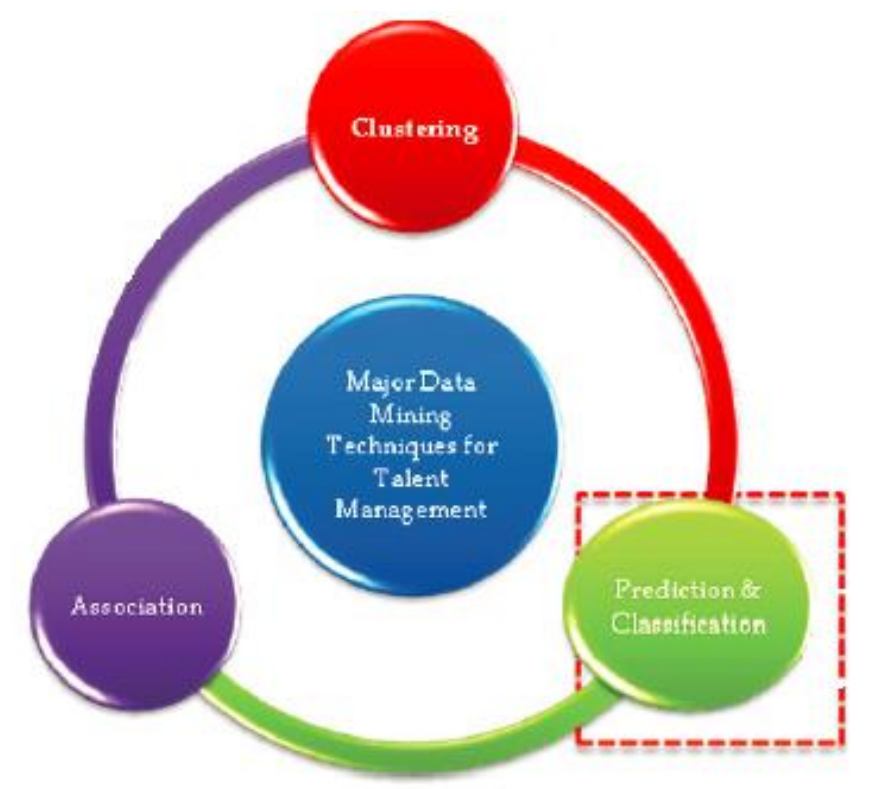

PICBE | 441

Figure 2. Data mining tasks for Talent Management

Source: Author's own research.

Setting up the experiment in this study has several tasks such as construction of simulated data, placing exceptional values, reducing attributes and determine model precision. However, due to difficulties to obtain real data from a human resources department because of privacy and security issues in order to explore, this study simulates human talent datasets using a data generator.

The first set of data comprises one hundred records (dataset1), while the second set of data contains a thousand records (dataset2). In many cases, the simulated data can be very close to your ideal and can produce such a good model for data mining. For this reason, in this study, I have inserted exceptional values in the first data set (dataset1) in order to avoid this problem and thus form a new set of data known as dataset3.

In this paper, selected and used classification techniques are based on common techniques used for classification and predictive data mining. As already mentioned, classification techniques are chosen, like neural networks, which are quite popular in the data mining community and used as a classification model. The decision tree approach known as "divide-and-conquer" consists of a set of independent courts for classification, while the nearest neighbor classification is used, which is based on metric distances.

Table 2. summarizes selected classification techniques using data, such as decision tree, neural networks and nearest neighbor. In this study, I tried to use C4.5 and random forests for the decision tree category, multilayer perception and radial basis functions network for the neural networks category and K-Star for the nearest neighbor category.

Table 2. - Classification techniques

\begin{tabular}{|l|ll|}
\hline \multicolumn{1}{|c|}{$\begin{array}{c}\text { Data mining } \\
\text { techniques }\end{array}$} & \multicolumn{1}{c|}{ Classification algorithms } \\
\hline Decision tree & $\begin{array}{l}\text { C4.5 - Induction decision tree - the objective is nominal, and entries } \\
\text { can be registered or interval. Sometimes the size of trees is reduced } \\
\text { significantly induced when cutting adopt a different strategy. }\end{array}$ \\
- $\begin{array}{l}\text { Random forest - Chooses a test based on a number of random } \\
\text { features at each node, carrying out cuts. Random Forest is building a } \\
\text { lot of random trees by wrapping assemblies. }\end{array}$ \\
\hline
\end{tabular}




\begin{tabular}{|l|l|}
\hline Neural networks & $\begin{array}{l}\text { Multi-Layer Perception - An accurate predictor for basic problems of } \\
\text { classification. Given a fixed network structure, should establish } \\
\text { appropriate weights for network connections }\end{array}$ \\
$\qquad \begin{array}{l}\text { Radial basis function network - Another popular type of power } \\
\text { supply, which has two layers, without counting the input layer, and a } \\
\text { multilayer perceptron different in the way of hidden units carrying } \\
\text { out calculations. }\end{array}$ \\
\hline Nearest neighbor k-NN & $\begin{array}{l}\mathrm{K}^{*}-\text { An example based on learning using distance metric to measure } \\
\text { the similarity of courts and general function of the distance-based } \\
\text { transformation. }\end{array}$ \\
\hline
\end{tabular}

The data sets contain information on a person's knowledge and skills, knowledge management, individual qualities and professional evaluation. For knowledge and skills, it wore generated values for 16 variables, while for knowledge management, individual qualities and professional evaluation, just 8 variables. These data sets were processed using Weka. After analyzing data sets, the following results were obtained, rounded to two decimal places:

Table 3. - Obtained results on simulated data sets

\begin{tabular}{|l|l|l|l|}
\hline Classification algorithm & Dataset1 & Dataset2 & Dataset3 \\
\hline Rrandom forest & 66,81 & 89,46 & 59,72 \\
\hline $\mathrm{K}^{*}$ Star & 79,30 & 86,04 & 62,29 \\
\hline Multi-layer perception & 77,12 & 92,63 & 74,94 \\
\hline Rradial basis function network & 70,56 & 94,28 & 80,72 \\
\hline C4.5 & 86,35 & 96,18 & 82,77 \\
\hline
\end{tabular}

Source: Author's own research.

The result for the full attributes shows that when we use more data (Dataset2) in training, a model can be developed with greater accuracy. Apart from that, the accuracy for Dataset3 containing outliers is less for all classifiers demonstrating the effect of outliers in

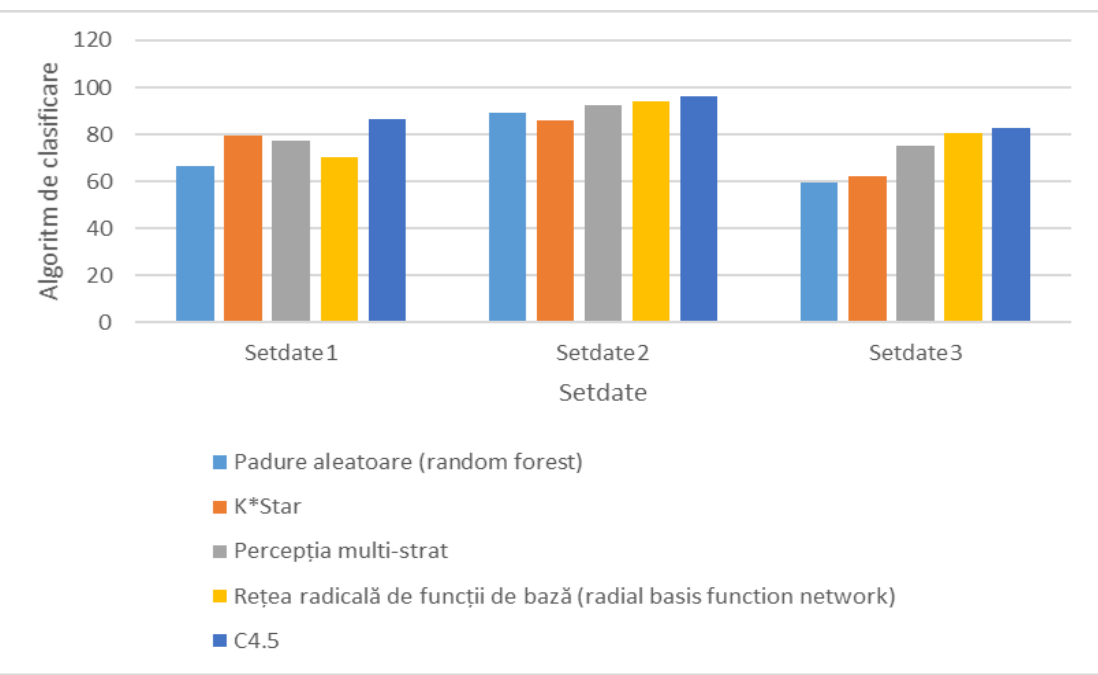

the data set accuracy of the model.

Figure 3. Model accuracy when using all attributes

Source: Author's own research.

The second phase of the experiment is to provide a relevant analysis in order to determine the accuracy of selected classification using the attribute sets of data reduction. In this experiment, I focused on datasets 1 and 2 . The purpose of this attribute reduction 
process is to select the most relevant data set attribute. The process of reduction is implemented with the aid of Boolean logic. By reducing attribute, we can reduce the time and space preprocessing and processing. The following table presents analysis results relevant to attribute reduction, five attributes being selected. Using these variables reduction attributes, the second stage of the experiment is implemented. The purpose of this experiment is to find out precision grading techniques with a reduction attribute attributes using the shortest length and combination of attributes importance after the reduction.

Table 4. - Result obtained when reducing attributes

\begin{tabular}{|l|l|l|}
\hline Classification algorithm & Setdate1 & Setdate2 \\
\hline Rrandom forest & 60,17 & 64,87 \\
\hline K*Star & 62,39 & 65,34 \\
\hline Multi-layer perception & 57,83 & 62,19 \\
\hline Rradial basis function network & 61,24 & 66,55 \\
\hline C4.5 & 63.17 & 65.43 \\
\hline
\end{tabular}

Source: Author's own research.

For this experiment, the result shows that the use of multiple attributes will affect accuracy. Therefore, this result shows that most of the attributes of the data set are important and should be taken into account. However, a combination of attributes of the reduction in the second stage of the experiment, the accuracy of the classifier is greater than the length of the shortest attributes (attribute reduction datasets).

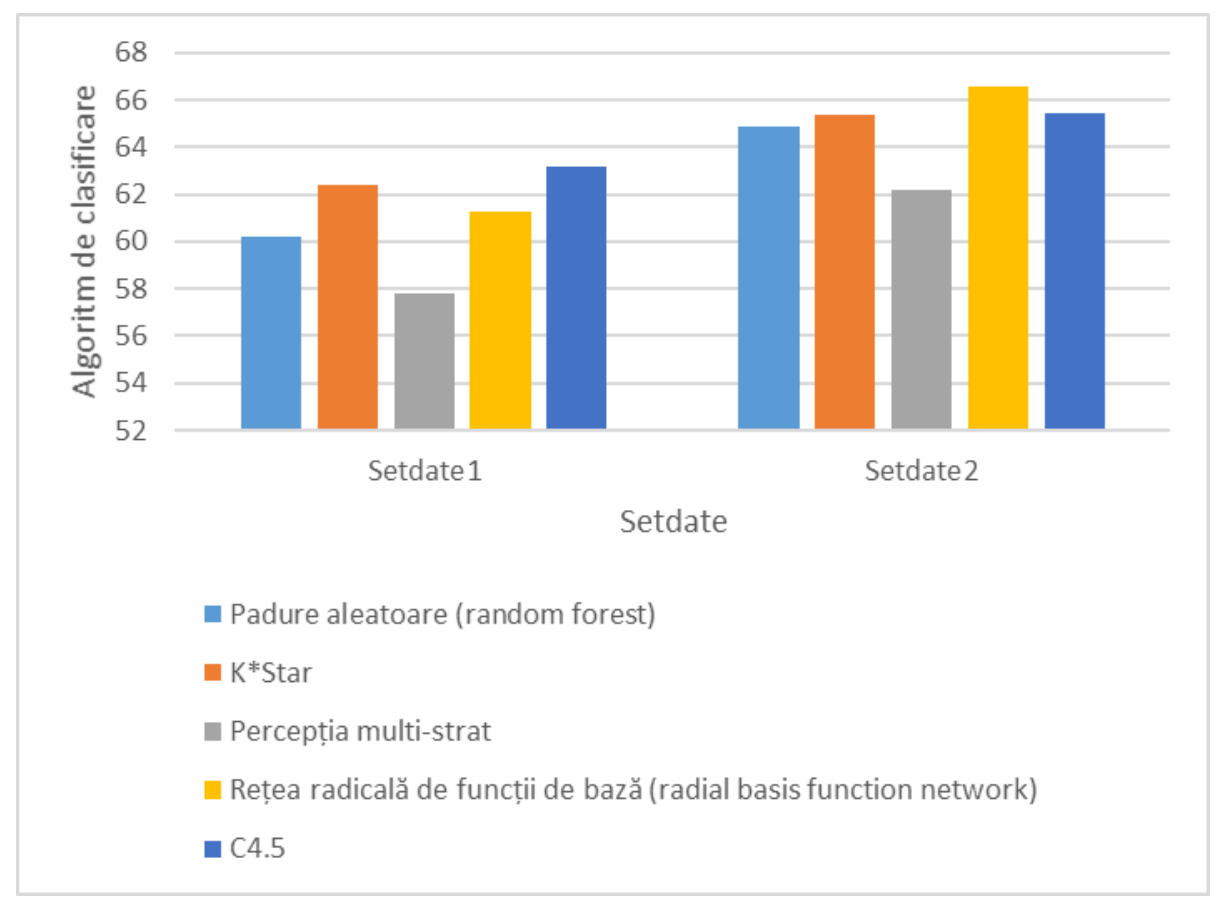

Figure 4. Model accuracy when reducing attributes

Source: Author's own research.

Table 5 shows the accuracy for the main attributes for datasets 1 and 2. C4.5 classifier has the highest precision for both sets of data at this stage of the experiment. 
Table 5. - Result obtained with attributes importance

\begin{tabular}{|l|c|c|}
\hline Classification algorithm & Setdate1 & Setdate2 \\
\hline Rrandom forest & 83,50 & 94,86 \\
\hline K*Star & 75,38 & 97,44 \\
\hline Multi-layer perception & 77,12 & 96,63 \\
\hline Rradial basis function network & 74,39 & 98,06 \\
\hline C4.5 & 92,61 & 99,13 \\
\hline
\end{tabular}

The following figure shows the accuracy of the data sets for the two models, attribute reduction and attribute importance, in the second stage of the experiment.

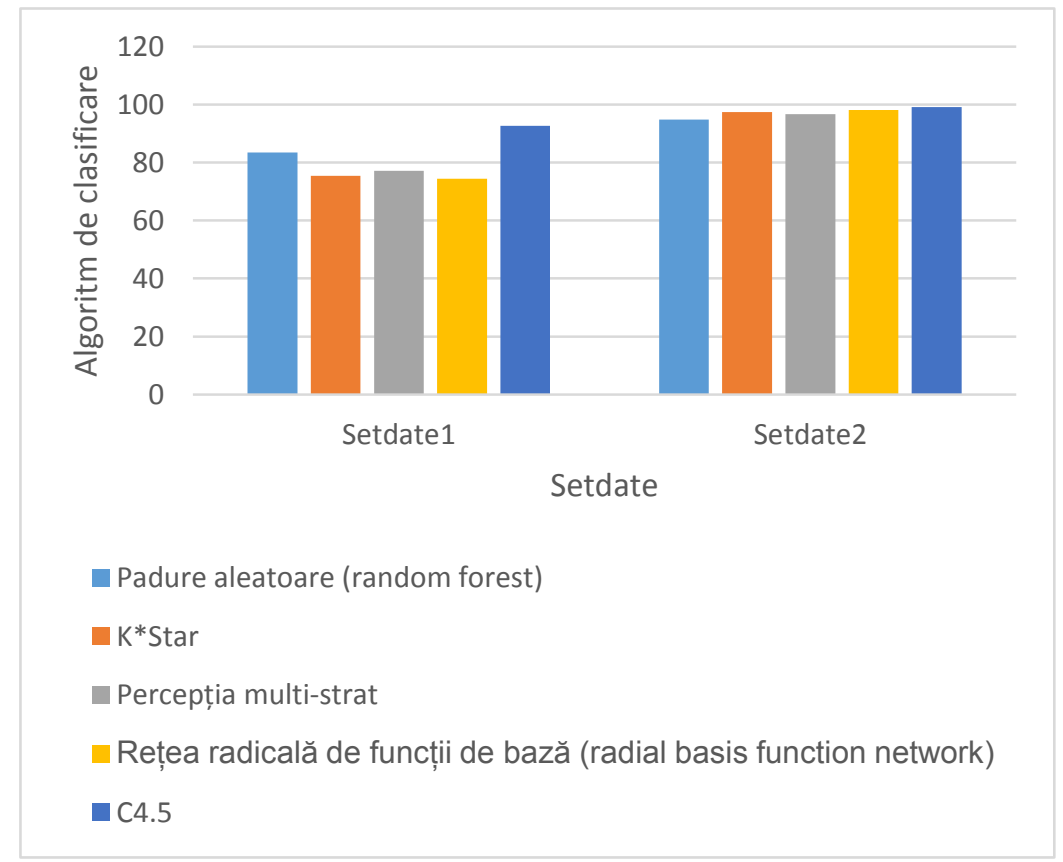

Figure 5. Model accuracy with attributes importance

Source: Author's own research.

\section{Proposing a prototype}

Based on the results obtained, a prototype can be developed. Thus, Weka was used to calculate the weights for all attributes depending on the functions of the company. For determining the ratios, it data had to be formatted properly, as required Weka.

As it can be seen in Figure 5, the program has provided us in decreasing order of importance, the attributes and their weight.

These weights were manually entered into the created database, and based on them a score will be calculated for each candidate using the following formula:

$$
\text { Score }=\frac{\sum_{i=1}^{16} C A_{i} * P C A_{i}+\sum_{i=1}^{8} C M_{i} * P C M_{i}+\sum_{i=1}^{8} C I_{i} * P C I_{i}+\sum_{i=1}^{8} E P_{i} * P E P_{i}}{\sum_{i=1}^{16} C A_{i}+\sum_{i=1}^{8} C M_{i}+\sum_{i=1}^{8} C I_{i}+\sum_{i=1}^{8} E P_{i}}
$$

Thus, using the previous formula (1), the scores of candidates are determined and plotted to allow the user to easily interpret the result of calculation and differences between candidates. Also, depending on the selected function, a plot showing similarities to 
those employed in the company, to highlight the differences between them and employees and candidates.

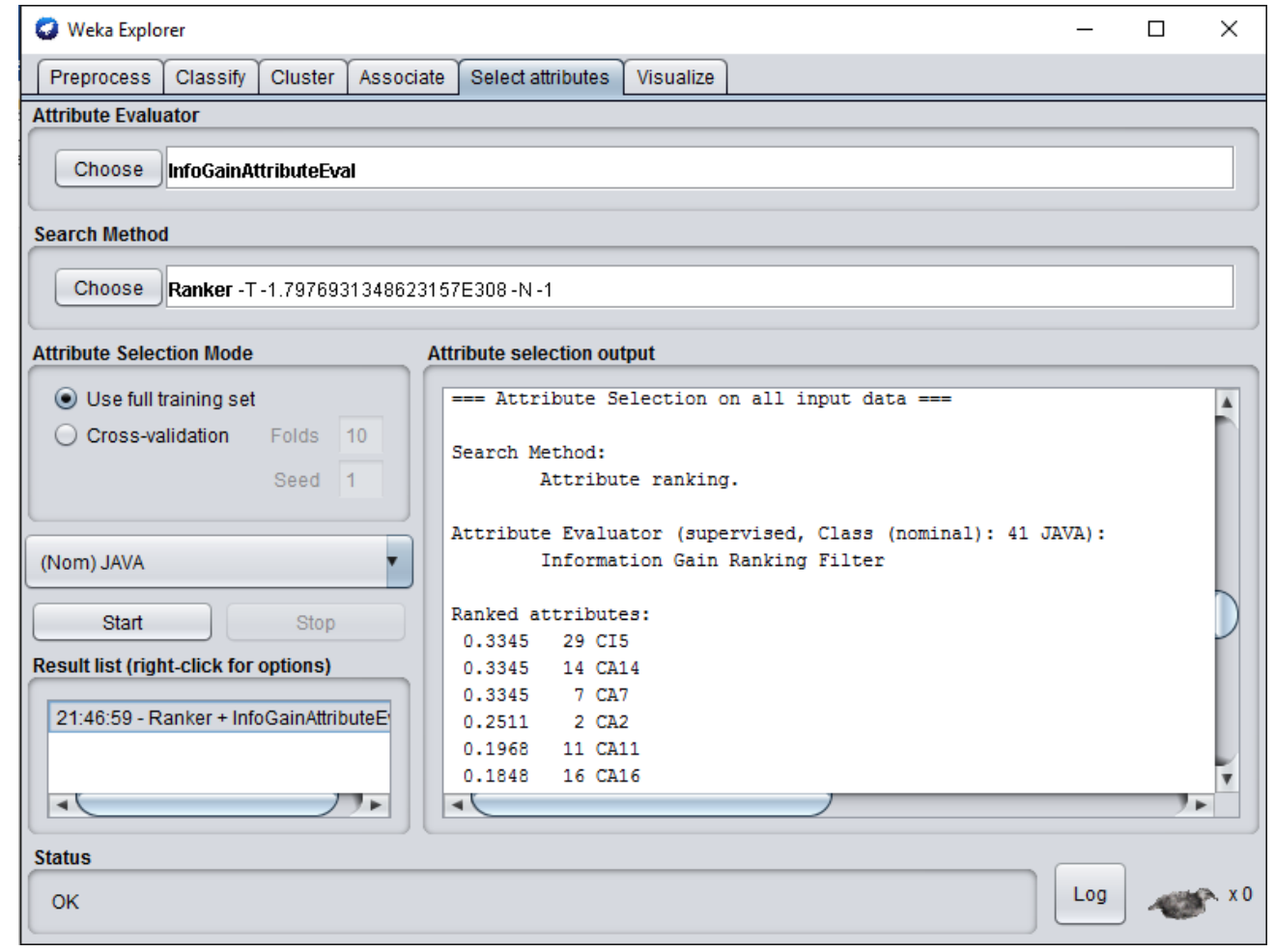

PICBE | 445

Figure 6. Attribute selection in Weka

Source: Author's own research.

\section{Potential impact}

Implementation of the prototype will have a major impact and influence to facilitate access to relevant information supporting decisions managers recruiting staff by minimizing the selection process time required by offering rapid discovery of key information about candidates and improving the relevance of the information on which managers make decisions recruitment.

\section{Conclusions}

For more companies, the responsibility of employing lies with an algorithm always increasing in complexity. Many more factors are considered so that candidates come not knowing what to expect. Jobs that were once occupied on the basis of history, and in interviews, are now taken as a result of personality tests and data analysis because employers want to be as sure that a future employee is suitable for the role. Under pressure to reduce costs and increase productivity, employers try to predict specific results.

Although employment is an important business function, conventional methods are not sufficiently demanding. Depending on who decides the factors that transform a candidate in an employee can vary greatly (academic achievement, experience, physical appearance, personality). 
However, using too specific criteria for selecting candidates may expose the company risks of infringement of equal opportunities. Even if unintentionally, selection criteria exclude older people or minorities, it is enough reason that can expose the company to a dispute.

The paper shows a way in which the candidates can be evaluated and marked on an algorithm. Such a ranking method can be implemented in HR Systems to offer managers an insight on whether the new candidate is better or worse than a specific employee. The method can also be used to evaluate whether an employee / candidate is suited better on a different job role than the one it has / targets.

Also, the paper shows which classification algorithm (random forest, $\mathrm{K}^{*}$ Star, multilayer perception, radial basis function network or $\mathrm{C4.5}$ ) suits best for gaining valuable knowledge for human resource data. It is also shown how the number of records from a data set or the abnormal values influence the accuracy. Based on the results obtained, the C4.5 classification algorithm suited best for this study's scenario.

Managers who rely on their instinct may sometimes obtain desired candidate, but their suspicions were generally little value in predicting how the candidate would handle the work. Companies recommended to perform a statistical approach say that employment can improve results by reducing biases influence a manager.

\section{References}

ADP Research Institute: Harnessing Big Data: The Human Capital Management Journey to Achieving Business Growth - ADP Global Human Capital Management Decision Makers Survey, 2015.

Bloomberg Businessweek, September 13 - September 19, 2010 issue, 54.

Chien, C.F., \& Chen, L.F. (2008). Data mining to improve personnel selection and enhance human capital: A case study in high-technology industry. Expert Systems and Applications, 34(1), 380-290.

Chen, K.K., Chen, M.Y., Wu, H.J., \& Lee, Y.L. (2007). Constructing a Web-based Employee Training Expert System with Data Mining Approach. Paper presented at the Paper in The 9th IEEE International Conference on E-Commerce Technology and The $4^{\text {th }}$ IEEE International Conference on Enterprise Computing, E-Commerce and Eservices (CEC-EEE 2007).

Elizabeth G. Chambers, Mark Fouldon, Helen Handfield-Jones, Steven M. Hankin, Edward G. Michaels III 1998, The War for Talent.

Han, J. \& Kamber, M. (2001). Data mining: concepts and techniques (Morgan-Kaufman Series of Data Management Systems), Academic Press, San Diego.

Huang, M.J., Tsou, Y.L., \& Lee, S.C. (2006). Integrating fuzzy data mining and fuzzy artificial neural networks for discovering implicit knowledge. Knowledge-Based Systems, 19(6), 396-403.

Ranjan, J. (2008). Data Mining Techniques for better decisions in Human Resource Management Systems. International Journal of Business Information Systems, 3(5), 464-481.

Tai, W.S., \& Hsu, C.C. (2005). A Realistic Personnel Selection Tool Based on Fuzzy Data Mining Method. Retrieved from www.atlantispress.com/php/download_papaer?id=46, 9/1/2008. 
Tung, K.Y., Huang, I.C., Chen, S.L., \& Shih, C.T. (2005). Mining the Generation Xer's job attitudes by artificial neural network and decision tree - empirical evidence in Taiwan. Expert Systems and Applications, 29(4), 783-794.

Tso, G.K.F., \& Yau, K.K.W. (2007). Predicting electricity energy consumption: A comparison of regression analysis, decision tree and neural networks. Energy, 32, 1761-1768.

Zhao, X. (2008). An Empirical Study of Data Mining in Performance Evaluation of HRM. Paper PICBE | 447 presented at the International Symposium on Intelligent Information Technology Application Workshops, Hangzhou, China. 